\title{
Successful treatment of anti-NMDA receptor encephalitis with a prompt ovarian tumour removal and prolonged course of plasmapheresis: A case report
}

\author{
ELZBIETA RYPULAK ${ }^{1}$, MICHAL BORYS ${ }^{1}$, PAWEL PIWOWARCZYK ${ }^{1}$, MAGDALENA FIJALKOWSKA $^{1}$, \\ BEATA POTREC ${ }^{1}$, JUSTYNA SYSIAK ${ }^{1}$, JANUSZ SPUSTEK ${ }^{2}$, ALICJA BARTKOWSKA-SNIATKOWSKA ${ }^{3}$, \\ JAN KOTARSKI $^{4}$, WALDEMAR A. TURSKI ${ }^{5}$, KONRAD REJDAK $^{6}$ and MIROSLAW CZUCZWAR ${ }^{1}$
${ }^{1}$ The Second Department of Anaesthesiology and Intensive Therapy, Medical University of Lublin, Lublin; ${ }^{2}$ Department of Neurology, Neuropsychiatric Hospital; ${ }^{3}$ Department of Paediatric Anaesthesiology and Intensive Therapy, Poznań University of Medical Sciences, Poznań; Departments of ${ }^{4}$ Gynaecological Oncology and Gynaecology, \\ ${ }^{5}$ Experimental and Clinical Pharmacology, and ${ }^{6}$ Neurology, Medical University of Lublin, Lublin, Poland
}

Received June 3, 2016; Accepted October 10, 2016

DOI: $10.3892 / \operatorname{mco} .2016 .1054$

\begin{abstract}
Anti-N-methyl-d-aspartate-receptor (NMDAR) encephalitis is an uncommon autoimmune disorder with a wide spectrum of neuropsychiatric symptoms. There is a great requirement to emphasize the importance of a multidisciplinary team approach in the process of diagnosis and treatment of the potentially fatal condition, including psychiatrists, neurologists, gynaecologists and intensivists. Physicians must be aware that psychiatric and neurological disorders, which are typical features for NMDAR encephalitis in young women with ovarian tumours, may progress into status epilepticus and respiratory insufficiency. This disease can only be successfully treated with prompt surgical intervention and an early implementation of a wide array of immunosuppressive therapies. Optimal timing of initiation of therapeutic plasma exchange, as well as duration of treatment necessary to achieve desirable outcomes in patients with NMDAR remains unknown. The present case report aims to raise awareness about the importance of early implementation of this potentially life-saving therapy and continuing the treatment courses until full subsidence of symptoms.
\end{abstract}

\section{Introduction}

Anti-N-methyl-D-aspartate-receptor (NMDAR) encephalitis is an autoimmune disorder with a wide spectrum of neuropsychiatric symptoms, which was first described in

Correspondence to: Professor Miroslaw Czuczwar, The Second Department of Anaesthesiology and Intensive Therapy, Medical University of Lublin, ul. Jaczewskiego 8, 20-090 Lublin, Poland E-mail: czuczwarm@gmail.com

Key words: anti-NMDA receptor antibodies, autoimmune encephalitis, therapeutic plasma exchange, status epilepticus, EEG abnormalities
2005 by Vitalini et al (1). It has recently been demonstrated that this potentially fatal disease is significantly underdiagnosed and may contribute to the majority of cases of non-infectious encephalitis of previously unknown aetiology (2). Vitalini et al (1) retrospectively analysed >500 cases of intensive care unit (ICU) admission and identified 7 patients with encephalitic signs (psychiatric and cognitive disturbances, as well as focal or generalized seizures progressing into status epilepticus), cerebrospinal fluid (CSF) inflammation and exclusion of bacterial or viral infection, and in 6 cases the presence of anti-NMDAR antibodies was confirmed. A positive serum or CSF sample screening for antibodies to the NMDAR subunit remains the gold-standard in diagnosing the disease and must be performed in all patients presenting with an acute onset of psychiatric symptoms with atypical features or unusual movements. However, emerging data exists to suggest that electroencephalograms (EEG) can also be very useful in aiding clinicians to diagnose anti-NMDAR encephalitis (3).

Over the past years, increasing awareness of autoimmune mediated forms of encephalitis with antibodies against neuronal surface antigens have led to an improvement in prognosis, predominantly due to wider application of immunomodulatory therapies. Notably, despite major progress in understanding the pathophysiology of anti-NMDAR encephalitis, there remains a clear requirement for a good quality data regarding the optimal treatment of the disease, predominantly since the type of immunotherapy that is most effective in controlling the symptoms of the disease remains a matter of debate. Numerous patients, particularly with a prolonged or severe form of the disease, do not respond to first-line immunotherapy [steroids or intravenous immunoglobulins (IVIg)] and may require therapeutic plasma exchange (TPE), which is commonly used to treat a number of neurological conditions, including Guillain-Barré syndrome, myasthenia gravis, chronic inflammatory demyelinating polyneuropathy, Lambert-Eaton syndrome, multiple sclerosis, neuromyelitis optica, paraproteinemic polyneuropathy, Sydenham's chorea and natalizumab-associated progressive 
multifocal leukoencephalopathy (4). Despite the increasing number of potential indications for TPE in the treatment of neurological disorders, the proven efficacy, side effects and costs must be taken into consideration prior to a decision being made to implement this therapy.

\section{Case report}

A 23-year-old female was admitted to a psychiatric ward, presenting with acute confusion, agitation and behavioural changes. The initial diagnosis was the first episode of schizophrenia or schizoaffective disorder. Her past medical history was non-significant and no prior psychiatric or psychological problems were reported. An episode of an upper respiratory tract infection, which preceded the psychotic symptoms by few days was notable. Upon admission, agitation and restlessness were observed, followed by progressive mutism and somnolence, which were the predominant symptoms of the disease within 5 days. The patient was administered standard antipsychotic treatment during her entire stay in the psychiatric ward, which included haloperidol, olanzapine and aposulpiryd, without any improvement in her psychiatric condition. On day 5 , the patient's neurological condition significantly deteriorated; decreased level of consciousness and loss of muscle tone were observed. Involuntary movements of upper limbs, jaw and eyes were also noticed, as well as clonic seizures, which were treated with intravenous diazepam. The initial diagnosis was changed to infectious encephalitis and the patient was transferred to a neurological ward at the state hospital, where CSF and blood samples were obtained and imaging studies of the brain, as well as EEG were performed (Fig. 1).

Results of the CSF analysis revealed a lymphocytic pleocytosis (60 white cells $/ \mu \mathrm{l})$, a normal protein level $(28.3 \mathrm{mg} / \mathrm{dl}$; normal value range: $15-45 \mathrm{mg} / \mathrm{dl}$ ) and a normal glucose level (81 mg/dl). EEG recordings revealed generalized rhythmic delta activity with superimposed rhythmic beta frequency activity ('extreme delta brush') (5). Computed tomography (CT) of the head revealed no pathological changes. The magnetic resonance imaging (MRI), which was performed upon admission to the neurological ward, revealed only two hyperintense lesions in subcortical white matter in the frontal lobes on T2/FLAIR. Additionally, initial focal seizures evolved into generalized tonic-clonic repetitive seizures, which did not respond to various anticonvulsants, including clonazepam, sodium valproate, phenytoin and carbamazepine. Subsequently, treatment with acyclovir was initiated for presumptive viral encephalitis. Immunotherapy was also implemented and the patient received a daily dose of $20 \mathrm{~g}$ of IVIg for 3 consecutive days. The CSF antibody tests were negative for Epstein-Barr virus, cytomegalovirus, human immunodeficiency virus, herpes simplex virus and varicella-zoster virus. The patient's condition deteriorated further; she developed a refractory status epilepticus with concomitant respiratory failure and autonomic instability and was referred to a tertiary ICU on the 27 th day of hospitalization.

The clinical course and the treatment protocol are summarized in Fig. 2. Upon admission to the ICU, the patient was comatose with a Glasgow Coma Scale (GCS) score of 6 . Neurological examination revealed that the patient exhibited spontaneous and provoked involuntary movements of the eyes, jaw, and upper and lower limbs, despite receiving a constant infusion of clonazepam and thiopental. Excessive salivation was also noted. The patient's trachea was intubated and mechanical ventilation was initiated. Sedatives (propofol, midazolam) and opioids (remifentanil) were given in continuous infusion to facilitate mechanical ventilation, yet orofacial and limb dyskinesia were still present and difficult to control despite increasing infusion rates of propofol and midazolam. The subsequently obtained test results of CSF and blood were irrelevant with only slightly elevated white bloodcell count. Results of both blood and CSF cultures remained negative, and repeated CT scan of the brain was also normal. Since the infectious background of the disease was unable to be confirmed, autoimmunological encephalitis was suspected and high dose corticosteroids (methyloprednisolone $1 \mathrm{~g}$ daily for 6 days, followed by prednisone $40 \mathrm{mg}$ twice daily) were administered, without any improvement in the patient's neurological status. On the 7th day of her stay in the ICU, a CT scan of the abdomen and the pelvis revealed an ovarian cyst. Additionally, a transvaginal ultrasound confirmed a left ovarian mass suggestive of a teratoma. Laparoscopic oophorectomy was immediately performed. The blood and CSF samples were collected to search for neuronal surface antibodies and the anti-NMDAR antibodies were identified in the CSF (titers, 1:3.2), but not in the plasma. The diagnosis of anti-NMDAR encephalitis was made based on the above results. Two days later, on the 36th day of hospitalization, TPE was initiated and after the third course, the patient's neurological condition markedly improved (an increase of GCS score from 6 to 11). She gradually started to open her eyes, obey simple commands and answer uncomplicated questions. Additionally, involuntary movements disappeared, yet salivation, autonomic instability and muscle weakness were still observed. The courses of TPE were continued to a total of 13 courses during her stay in the ICU. Meanwhile, another course of supplementary immunotherapy with IVIg (20 g every second day) was implemented due to its proven position as a first line treatment in the armamentarium against autoimmunological encephalitis. The relatively high frequency of TPE resulted in haemodynamic instability and coagulopathy, which required plasma transfusion; therefore, the intervals between TPE were increased from 1 to 3 days and the anticoagulant was changed from unfractionated heparin to citrate. The autonomic dysfunction persisted despite obvious improvement in cognitive status and involved severe bradycardia and hypersalivation, which made all the tracheal extubation efforts futile. Therefore, on the 25th day of the ICU stay, a tracheostomy was performed. The patient was subsequently weaned from mechanical ventilation and transferred to a neurological ward to continue intensive rehabilitation. She was discharged in a good clinical condition nearly 3 months after the onset of the disease. The follow-up assessment was performed 3 months following discharge. The patient's neurological state was tested with modified Rankin Scale (mRS) and she gained 1 point. She reported no significant disability and was able to perform all usual activities. The only neurological sequel included parasomnia and complete loss of memory during the entire hospitalization period.

\section{Discussion}

The present case study described a clinical course of anti-NMDAR encephalitis, which is one of the central nervous 


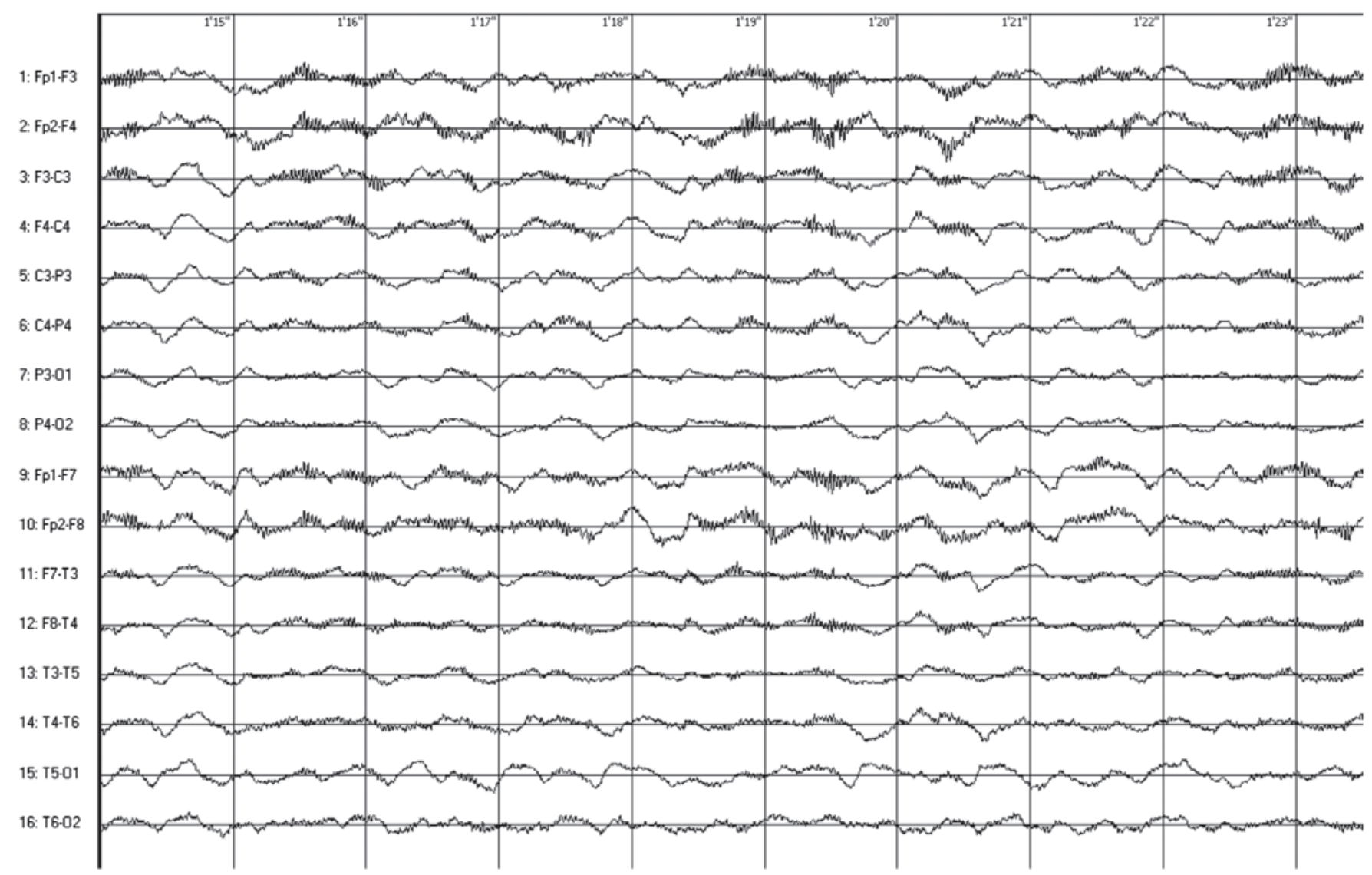

Figure 1. Encephalogram demonstrates generalized rhythmic delta activity with superimposed rhythmic beta frequency activity (extreme delta brush) obtained following 1 week of hospitalisation.

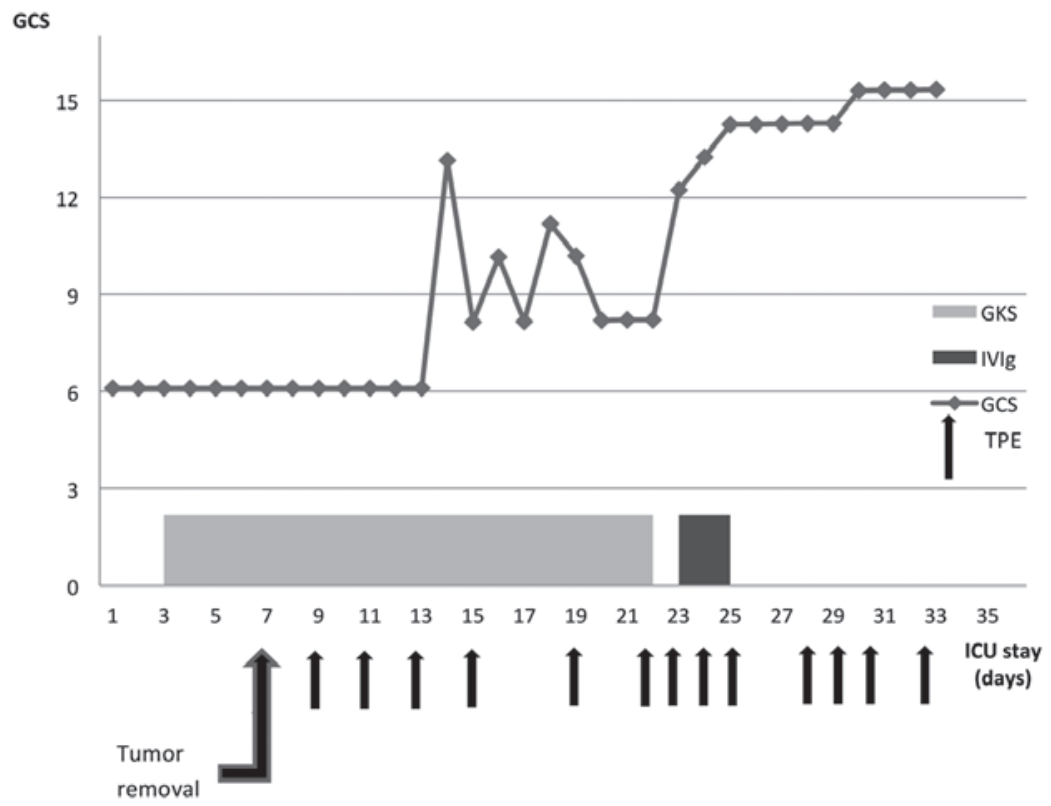

Figure 2. Clinical course of the patient and treatment protocol after the patient's referral to the ICU (beginning at 27th day of hospitalisation). ICU, intensive care unit; GCS, Glasgow Coma Scale; GKS, IVIg, intravenous immunoglobulins; TPE, therapeutic plasma exchange.

system (CNS) diseases, where the presence of the neuronal surface antibodies can be demonstrated (6). This relatively uncommon disorder has a typical sequential presentation: sudden onset with prodromal, fever-like symptoms, followed by a psychiatric disorders, decreased level of conscious accompanied by intractable focal and clonic seizures, dyskinesias and autonomic instability (7). In the present case, all of the above-mentioned symptoms were observed, so the clinical 
course of the anti-NMDAR encephalitis was typical. In addition, the patient was a young female, which is also a common feature of this type of autoimmunological encephalitis, since this patient population accounts for $\sim 80 \%$ of reported cases (8). Furthermore, the coexistence of an ovarian tumour, which was confirmed to be a teratoma in this case, is also a typical for antiNMDAR encephalitis. This type of encephalitis is suggested to be strongly associated with malignancies, including ovarian, mediastinal and testicular teratomas, Hodgkin's lymphoma, small cell lung carcinoma, and neuroblastomas. However, more recent studies have challenged these data showing that up to $80 \%$ of patients may present with a non-paraneoplastic type of the disease, making the diagnosis even more difficult (9). When anti-NMDAR is suspected the diagnostic tests typically include detection of NMDAR autoantibodies in CSF and/or in the serum as the core of diagnosis, predominantly because other laboratory tests and imaging studies of the brain appear to be irrelevant $(10,11)$. On the other hand, MRI scans of the patient's CNS revealed multifocal subcortical white matter lesions in T2/FLAIR, which were also described by other authors and may be concomitant with anti-NMDAR encephalitis (9).

There is ongoing debate as to whether serum or CSF must be tested for in the presence of anti-NMDAR antibodies. In the present case, high levels of anti-NMDAR antibodies were detected in the CSF, but not in the plasma. The association between the prodromal flu-like symptoms and the antibodies against NMDAR is also a matter of debate. Certain authors emphasise the connection between a viral infection and damage of the blood-brain-barrier, which facilitates transfer of NMDAR autoantibodies to the CNS, whilst others claim that flu-like symptoms are only a part of an early immunological activation (12). Nevertheless, when anti-NMDAR encephalitis is suspected it appears absolutely crucial to search for the antibodies in both serum and CSF, yet other more readily available diagnostic measures must also be implemented in order to obtain the final diagnosis. In the present case, a retrospective analysis of the first EEG recordings revealed an unique electrographic pattern, which is referred to as "extreme delta brush", due to of its resemblance to waveforms observed in premature infants (13). Although the specificity of this finding remains unclear, its presence is usually associated with a more prolonged and severe course of illness. An EEG maybe extremely valuable in distinguishing between encephalitis and a primary psychiatric disorder, since a vast majority of patients with anti-NMDAR encephalitis exhibit a non-specific slowing at a certain stage during the illness. In addition, characteristic pattern of generalized rhythmic delta activity with superimposed rhythmic beta frequency activity may appear, which was described in a large series of patients unique to this disorder (8).

Treatment of anti-NMDAR encephalitis remains challenging, since no comprehensive guidelines have been published to date. The majority of authors agree that treatment must target both the cause and the clinical consequences of the encephalitis. It is generally accepted that the anti-NMDAR encephalitis treatment is much more effective in patients who have an underlying tumour removed and there are numerous cases described in which ovarian teratomas were discovered years after initial onset of symptoms, particularly in patients who experienced a slow recovery (14). Certain authors advocate early oophorectomy even in cases when the presence of an ovarian tumour cannot be confirmed in imaging studies. In a case described by Peery et al (14), postoperative biopsy revealed an occult teratoma, and the surgical removal of the tumour resulted in an improvement of clinical symptoms. In the majority of cases, immunotherapy is the first-line treatment and typically includes corticosteroids, IVIg and TPE, administered alone or in combination (15). The second-line treatment includes rituximab and cyclophosphamide, and is used predominantly in patients who either exhibit a delayed diagnosis or did not have an underlying malignancy (16). It is worth mentioning that the role of specific treatment options remains a matter of debate and numerous authors provide conflicting data on the subject, with special regards to the role of TPE, which in the opinion of Dalmau et al (17) should not be used routinely. On the other hand, numerous recently published case studies, where implementation of TPE was associated with an improvement of clinical status, exist $(18,19)$. In a recent paper published by DeSena et al $(20)$, the efficacy of intravenous methylprednisolone administered alone or following TPE was compared in the treatment of anti-NMDAR receptor encephalitis. The obtained results were clearly in favour of administering steroids only after TPE was performed in the studied patient population.

In the present case, the patient was initially treated in the neurological ward with the use of corticosteroids (methylprednisolone) and IVIg due to suspected infectious encephalitis. This first-line immunosuppressive treatment failed to produce any improvement of the patient's clinical condition. Similarly, the second course of high-dose corticosteroids administered in the ICU was not effective. The patient's clinical condition only improved following the third course of TPE, which is in line with the case study published by Nunez-Enamorado et al (21). By contrast, clinical improvement in cases of anti-NMDAR encephalitis is not always achieved with TPE $(22,23)$. According to the guidelines published by the American Society for Apheresis, the use of TPE must be considered as a third-line treatment in paraneoplastic neurological syndromes (grade 2C) (24).

In conclusion, NMDAR encephalitis is a potentially reversible disorder with a good clinical outcome if diagnosed and treated promptly. A multimodal immunosuppresive therapy may result in an improvement in neurological symptoms in $>60 \%$ of patients (25). In the present case, a long-term followup examination revealed a good clinical outcome, without any major neurological or psychiatric complications following the anti-NMDAR encephalitis. A multidisciplinary team, including psychiatrists, neurologists and intensivists, must be involved in the process of recognition and management of the disease. The gold standard for diagnosing anti-NMDAR encephalitis is still detecting the antibodies in either the CSF or plasma; however, other diagnostic measures, including EEG or MRI may also aid clinicians in obtaining the final diagnosis. Tumour removal and pharmacotherapy remain the first-line treatment in the majority of cases, yet TPE must also be considered in the clinician's armamentarium, particularly in cases where initial treatment has failed. Available data remains ambiguous and therefore there remains an urgency for good quality clinical trials. 


\section{References}

1. Vitaliani R, Mason W, Ances B, Zwerdling T, Jiang Z and Dalmau J: Paraneoplastic encephalitis, psychiatric symptoms, and hypoventilation in ovarian teratoma. Ann Neurol 58: 594-604, 2005.

2. Prüss H, Dalmau J, Harms L, Höltje M, Ahnert-Hilger G, Borowski K, Stoecker W and Wandinger KP: Retrospective analysis of NMDA receptor antibodies in encephalitis of unknown origin. Neurology 75: 1735-1739, 2010.

3. Barry H, Byrne S, Barrett E, Murphy KC and Cotter DR: AntiN-methyl-d-aspartate receptor encephalitis: Review of clinical presentation, diagnosis and treatment. BJPsych Bull 39: 19-23, 2015.

4. Gwathmey K, Balogun RA and Burns T: Neurologic indications for therapeutic plasma exchange: 2013 update. J Clin Apher 29: 211-219, 2014.

5. Schmitt SE, Pargeon K, Frechette ES, Hirsch LJ, Dalmau J and Friedman D: Extreme delta brush: A unique EEG pattern in adults with anti-NMDA receptor encephalitis. Neurology 79 1094-1100, 2012

6. Zuliani L, Graus F, Giometto B, Bien C and Vincent A: Central nervous system neuronal surface antibody associated syndromes: Review and guidelines for recognition. J Neurol Neurosurg Psychiatry 83: 638-645, 2012.

7. Granerod J, Ambrose HE, Davies NW, Clewley JP, Walsh AL, Morgan D, Cunningham R, Zuckerman M, Mutton KJ, Solomon T, et al; UK Health Protection Agency (HPA) Aetiology of Encephalitis Study Group: Causes of encephalitis and differences in their clinical presentations in England: A multicentre, population-based prospective study. Lancet Infect Dis 10: 835-844, 2010.

8. Titulaer MJ, McCracken L, Gabilondo I, Armangué T, Glaser C, Iizuka T, Honig LS, Benseler SM, Kawachi I, MartinezHernandez E, et al: Treatment and prognostic factors for long-term outcome in patients with anti-NMDA receptor encephalitis: An observational cohort study. Lancet Neurol 12: 157-165, 2013.

9. Irani SR, Bera K, Waters P, Zuliani L, Maxwell S, Zandi MS, Friese MA, Galea I, Kullmann DM, Beeson D, et al: N-methylD-aspartate antibody encephalitis: Temporal progression of clinical and paraclinical observations in a predominantly nonparaneoplastic disorder of both sexes. Brain 133: 1655-1667, 2010.

10. Dalmau J, Gleichman AJ, Hughes EG, Rossi JE, Peng X, Lai M, Dessain SK, Rosenfeld MR, Balice-Gordon R and Lynch DR: Anti-NMDA-receptor encephalitis: Case series and analysis of the effects of antibodies. Lancet Neurol 7: 1091-1098, 2008.

11. Motta E, Gołba A, Kazibutowska Z, Huć M and Stęposz A: Anti-NMDA receptor encephalitis-case report. Neurol Neurochir Pol 46: 288-293, 2012 (In Polish).

12. Hammer C, Stepniak B, Schneider A, Papiol S, Tantra M, Begemann M, Sirén AL, Pardo LA, Sperling S, Mohd Jofrry S, et al: Neuropsychiatric disease relevance of circulating anti-NMDA receptor autoantibodies depends on blood-brain barrier integrity. Mol Psychiatry 19: 1143-1149, 2014.

13. Iizuka T, Sakai F, Ide T, Monzen T, Yoshii S, Iigaya M, Suzuki K, Lynch DR, Suzuki N, Hata T, et al: Anti-NMDA receptor encephalitis in Japan: Long-term outcome without tumor removal. Neurology 70: 504-511, 2008.
14. Peery HE, Day GS, Doja A, Xia C, Fritzler MJ and Foster WG: Anti-NMDA receptor encephalitis in children: The disorder, its diagnosis, and treatment. Handb Clin Neurol 112: 1229-1233, 2013.

15. Mann AP, Grebenciucova E and Lukas RV: Anti-N-methylD-aspartate-receptor encephalitis: Diagnosis, optimal management, and challenges. Ther Clin Risk Manag 10: 517-525, 2014.

16. Florance NR, Davis RL, Lam C, Szperka C, Zhou L, Ahmad S, Campen CJ, Moss H, Peter N, Gleichman AJ, et al: Anti-N-methyl-D-aspartate receptor (NMDAR) encephalitis in children and adolescents. Ann Neurol 66: 11-18, 2009.

17. Dalmau J, Lancaster E, Martinez-Hernandez E, Rosenfeld MR and Balice-Gordon R: Clinical experience and laboratory investigations in patients with anti-NMDAR encephalitis. Lancet Neurol 10: 63-74, 2011.

18. Zhang Y, Gao D, Ye H and Su Y:[Safety of plasma exchange therapy in patients with anti-NMDA receptor encephalitis. Zhonghua Yi Xue Za Zhi 95: 1505-1508, 2015 (In Chinese).

19. Shahani L: Steroid unresponsive anti-NMDA receptor encephalitis during pregnancy successfully treated with plasmapheresis. BMJ Case Rep 2015 (apr29 1): 29, 2015.

20. DeSena AD, Noland DK, Matevosyan K, King K, Phillips L, Qureshi SS, Greenberg BM and Graves D: Intravenous methylprednisolone versus therapeutic plasma exchange for treatment of anti-N-methyl-D-aspartate receptor antibody encephalitis: A retrospective review. J Clin Apher 30: 212-216, 2015.

21. Nunez-Enamorado N, Camacho-Salas A, Belda-Hofheinz S, Cordero-Castro C, Simon-De Las Heras R, Saiz-Diaz R, Martinez-Sarries FJ, Martinez-Menendez B and Graus F: Fast and spectacular clinical response to plasmapheresis in a paediatric case of anti-NMDA encephalitis. Rev Neurol 54: 420-424, 2012 (In Spanish).

22. Mirza MK, Pogoriler J, Paral K, Ananthanarayanan V, Mandal S, Mazin A, Baron B and Richa E: Adjunct therapeutic plasma exchange for anti-N-methyl-D-aspartate receptor antibody encephalitis: A case report and review of literature. J Clin Apher 26: 362-365, 2011.

23. Ikeguchi R, Shibuya K, Akiyama S, Hino S, Kubo H, Takeda T, Shibata N and Yamamoto K: Rituximab used successfully in the treatment of anti-NMDA receptor encephalitis. Intern Med 51: 1585-1589, 2012.

24. SchwartzJ, Winters JL, Padmanabhan A,Balogun RA,Delaney M, Linenberger ML, Szczepiorkowski ZM, Williams ME, Wu Y and Shaz BH: Guidelines on the use of therapeutic apheresis in clinical practice-evidence-based approach from the Writing Committee of the American Society for Apheresis: The sixth special issue. J Clin Apher 28: 145-284, 2013.

25. Ehrlich S, Fassbender CM, Blaes C, Finke C, Günther A, Harms L, Hoffmann F, Jahner K, Klingel R, Kraft A, et al: Therapeutic apheresis for autoimmune encephalitis: A nationwide data collection. Nervenarzt 84: 498-507, 2013 (In German). 